\title{
TRANSLATION OF METAPHORISED TERMS (THE CASE OF PATENTS)
}

\author{
Natalya V. Sokolova \\ Perm National Research Polytechnic University, Perm, Russia
}

\begin{abstract}
The paper focuses on the aspects of translating specialised lexical units of technical texts. Metaphorised means of cognitive information, namely terms, are of particular interest within the framework of technical texts characterized by the presence of cognitive information, which is dominant in such texts. Our hypothesis is that modern technical patents registering copyright for specific latest inventions may contain terms formed as a result of the metaphoric transfer with the purpose of rendering the cognitive information. Drawing upon English-language patents presenting discoveries in the area of nanotechnology and nanomaterials and selected from PATENTSCOPE database of the World Intellectual Property Organisation by the continuous sampling method, this paper highlights the techniques of translating metaphorised terms into Russian. To achieve the set goal, the comparative and descriptive methods have been utilized. The analysis of patents and their Russian translations has revealed that the following methods have been used to translate metaphorised terms: metaphorisation, with the meaning transfer method being retained; remetaphorisation, with the meaning transfer method being changed; demetaphorisation; demetaphorisation or metaphorisation of one and the same metaphorised term. The research demonstrates that remetaphorisation is the least used technique. The practical value of this paper consists in the use of the terminology research outcomes for translation purposes and in translation theory and practice courses.

Key words: translation, term, metaphorised term, patent, cognitive information, metaphorisation, demetaphorisation, remetaphorisation.
\end{abstract}

Citation. Sokolova N.V. Translation of Metaphorised Terms (the Case of Patents). Vestnik Volgogradskogo gosudarstvennogo universiteta. Seriya 2, Yazykoznanie [Science Journal of Volgograd State University. Linguistics], 2018, vol. 17, no. 1, pp. 107-114. (in Russian). DOI: https://doi.org/10.15688/jvolsu2.2018.1.11

УДК 81’25:811.111

Дата поступления статьи: 16.10 .2017

ББК 81.18

Дата принятия статьи: 12.02.2018

\section{ПРИЕМЫ ПЕРЕВОДА \\ АНГЛОЯЗЫЧНЫХ МЕТАФОРИЗИРОВАННЫХ ТЕРМИНОВ (НА МАТЕРИАЛЕ ПАТЕНТОВ)}

\author{
Наталья Владимировна Соколова \\ Пермский национальный исследовательский политехнический университет, г. Пермь, Россия
}

\begin{abstract}
Аннотация. Статья посвящена проблемам перевода специальной лексики, функционирующей в технических текстах. Объектом исследования избраны метафоризированные термины как носители когнитивной информации, доминирующей в технических текстах. В качестве материала для изучения послужили размещенные в базе РАTENTSCOPЕ Всемирной организации интеллектуальной собственности (World Intellectual صُ Property Organisation) современные англоязычные технические патенты, удостоверяющие авторство на конкретные новейшие изобретения в области нанотехнологий и наноматериалов, и их переводы на русский язык. Для достижения поставленной цели - выявить и охарактеризовать приемы перевода метафоризированных терминов - применялись сопоставительный и дескриптивный методы. Установлено, что в качестве приемов перевода метафоризированных терминов использовались: 1) метафоризация с сохранением спосо() ба переноса значения, 2) реметафоризация со сменой способа переноса значения, 3) деметафоризация,
\end{abstract}


4) деметафоризация или метафоризация одного и того же метафоризированного термина, что способствует появлению синонимичных терминов в русском языке. В рамках проведенного исследования можно говорить о меньшей востребованности приема реметафоризации. Практическая ценность работы определяется возможностью использования результатов исследования терминов, созданных на основе метафорического переноса, в переводческой деятельности, а также в курсах теории и практики перевода.

Ключевые слова: перевод, термин, метафоризированный термин, патент, когнитивная информация, метафоризация, деметафоризация, реметафоризация.

Цитирование. Соколова Н. В. Приемы перевода англоязычных метафоризированных терминов (на материале патентов) // Вестник Волгоградского государственного университета. Серия 2, Языкознание. - 2018. T. 17, № 1. - C. 107-114. - DOI: https://doi.org/10.15688/jvolsu2.2018.1.11

\section{Введение}

В последние десятилетия метафора изучается не столько в качестве средства создания образности, сколько как когнитивный механизм работы нашего сознания, который находит свое отражение в естественном языке: «Для большинства людей метафора является инструментом поэтического воображения и риторических излишеств - частью какого-то особенного, а не повседневного языка. По этой причине множество людей считает, что они прекрасно обходятся без метафор. Вопреки этому мнению мы обнаружили, что метафора пронизывает нашу повседневную жизнь, причем не только язык, но и мышление и деятельность. Наша обыденная понятийная система, в рамках которой мы думаем и действуем, по сути своей метафорична» [Лакофф, Джонсон, 2004, с. 27].

О разграничении художественной метафоры и метафоры как средства познания и осмысления действительности писал еще В.В. Виноградов: «Языковые метафоры - это знаки группировки понятий в идеологической системе языка и запечатления бытийных связей в процессе завоевания мира. А метафоры художественной речи, особенно те, которые не проносятся, как метеор, по полю произведения, а тянут за собою, как комета, обрывки своих словесных сфер, - эти метафоры есть продукт чисто словесных сочетаний» [Виноградов, 1980, с. 248-249].

Современные исследователи обращаются к рассмотрению функционирования метафоры в нехудожественных текстах: публицистических [Шебеко, 2012], научно-технических [Лосева, 2015; Finatto, 2010], научно-популярных [Уткина, 2006] и т. д. Ученые описывают особенности перевода метафоризиро- ванных лексических единиц, вводимых авторами для репрезентации прагматически переработанного научного знания и не являющихся при этом терминами [У ткина, 2006; Шебеко, 2012; Finatto, 2010], а также непосредственно метафоризированные термины и предлагают их классификации по способам образования в исходном языке [Горохова, 2014; Лосева, 2015]. В частности, в статье О.М. Лосевой приводится подробная классификация терминов машиностроительной отрасли, образованных путем метафорического переноса на основании сходства с частями и функциями человеческого организма [Лосева, 2015, c. 120]. В статье Н.В. Гороховой тщательно анализируются морфологические и семантические способы образования метафоризированных терминов трубопроводного транспорта [Горохова, 2014].

При этом проблемы, связанные с переводом метафоризированных терминов, остаются недостаточно изученными.

\section{Материал и методы исследования}

Если рассматривать метафору как средство познания и осмысления действительности, то необходимо учитывать тот факт, что такая метафора может и должна нести не столько эстетическую и эмоциональную, сколько когнитивную информацию, или объективные сведения об окружающей действительности. Характер передаваемой информации становится значимым параметром при классификации текстов, ориентированной на перевод. Так, И.С. Алексеева в одной из своих работ приводит таблицу «Значимые признаки текста», в которой представлено убывание когнитивной информации в текстах разной стилевой отнесенности - от научных к ху- 
дожественным [Алексеева, 2008, с. 38]. В рамках научно-технических текстов с доминирующей в них когнитивной информацией особый интерес составляют ее метафоризированные носители, в частности метафоризированные термины.

Следует отметить, что в лингвистической литературе понятие «технический текст» традиционно толкуется с точки зрения экстралингвистической и стилистической доминанты научного стиля как «научно-технический». Так, в авторитетный «Стилистический энциклопедический словарь русского языка» под редакцией М.Н. Кожиной включена статья «Научный стиль» (СЭСРЯ, 2006, с. 244 247), однако статья, посвященная техническому тексту и его стилистическим и языковым особенностям, в нем не представлена.

Мы считаем, что при общности стилевых черт и средств их выражения, которой обладают научные и технические тексты, именно их сосуществование в противопоставлении друг другу, то есть дихотомия, позволяет выявить ключевые различия между ними (см. таблицу).

В таблице стилеобразующие факторы, стилевые черты и средства их выражения, свойственные научному тексту, представлены с опорой на сведения, приведенные в СЭСРЯ, а стилевые параметры технического текста выявлены нами.

Как видим, технические тексты имеют существенное отличие от научных: стилеобразующим фактором для них является опре- деленность, или конкретность, которая проявляется в стилевых чертах и в наборе языковых средств их выражения в тексте.

Одним из жанров технических текстов является патент, основная стилевая черта которого - определенность, или конкретность, противопоставлена обобщенной отвлеченности, или абстрагизации, научных текстов.

Мы предполагаем, что современные технические патенты, удостоверяющие авторство на конкретные изобретения, могут содержать термины, образованные с помощью метафорического переноса с целью передачи когнитивной информации. Цель нашего исследования - выявить приемы перевода на русский язык таких метафоризированных терминов. В качестве основных методов исследования используются сопоставительный и дескриптивный методы.

Материалом для изучения послужили патенты из базы PATENTSCOPE Всемирной организации интеллектуальной собственности, или ВОИС (World Intellectual Property Organisation). Отрасль нанотехнологий является междисциплинарной, отражающей новейшие достижения и открытия в области техники, что позволяет предположить наличие в патентах из сферы нанотехнологий новых, специфичных для данной отрасли терминов, которые могут быть образованы путем метафорического переноса.

Методом сплошной выборки были отобраны 15 патентов, относящиеся к области наноматериалов и нанотехнологий, а затем

\section{Стилевая характеристика научного и технического текста}

\begin{tabular}{|c|c|}
\hline НАУЧНЫЙ ТЕКСТ & ТЕХНИЧЕСКИЙ ТЕКСТ \\
\hline \multicolumn{2}{|c|}{ Экстралингвистические стилеобразующие факторы } \\
\hline Объективность & Объективность \\
\hline Отвлечение от конкретного и случайного & Наличие конкретного, отвлечение от случайного \\
\hline $\begin{array}{l}\text { Логическая доказательность и последовательность } \\
\text { изложения }\end{array}$ & $\begin{array}{l}\text { Логическая доказательность и последовательность } \\
\text { изложения }\end{array}$ \\
\hline \multicolumn{2}{|c|}{ Стилевые черты } \\
\hline Обобщенно-отвлеченность, или абстрагизация & Определенность, или конкретность \\
\hline Подчеркнутая логичность & Подчеркнутая логичность \\
\hline Точность & Точность \\
\hline \multicolumn{2}{|c|}{ Средства выражения } \\
\hline $\begin{array}{l}\text { Глагольные формы несовершенного вида настоящего } \\
\text { времени со значением настоящего вневременного для } \\
\text { описания свойств объекта }\end{array}$ & $\begin{array}{l}\text { Глагольные формы совершенного вида для описания } \\
\text { имевших место конкретных, определенных ситуаций, } \\
\text { проведенных испытаний и пр. }\end{array}$ \\
\hline $\begin{array}{l}\text { Страдательный залог, сложноподчиненные предложе- } \\
\text { ния, обобщенно-личные предложения, безличные пред- } \\
\text { ложения }\end{array}$ & $\begin{array}{l}\text { Страдательный залог, сложноподчиненные предложе- } \\
\text { ния, определенно-личные предложения }\end{array}$ \\
\hline Термины & Термины \\
\hline
\end{tabular}


использованные в них метафоризированные термины (всего 15 единиц). После того в текстах перевода этих патентов на русский язык были выявлены русскоязычные эквиваленты метафоризированных терминов и определены приемы перевода, использованные переводчиками.

\section{Результаты}

Англоязычные метафоризированные термины из области наноматериалов и нанотехнологий переведены на русский язык с использованием различных приемов.

\section{Метафоризация с сохранением спо- соба переноса значения (4 лексические единицы).}

(1) The channel layer includes a semiconductor layer and a graphene layer in direct contact with the semiconductor layer, and the graphene layer includes a plurality of graphene islands spaced apart from each other (Lee, 2017).

Канальный слой включает в себя полупроводниковый слой и графеновый слой в непосредственном контакте с полупроводниковым слоем, а слой графена включает в себя множество островков графена, расположенных на некотором расстоянии друг от друга (перевод ВОИС) (Ли, 2017).

Импульсом к созданию множества метафоризированных терминов в области нанотехнологий стало открытие двумерной формы углерода - графена (graphene). Двукомпонентный термин graphene island образован посредством метафорического переноса на основании сходства формы (остров, островок). В переводе такая метафоризация сохраняется.

\section{Реметафоризация со сменой способа} переноса значения (2 лексические единицы).

(2) The quantum dots 7 may comprise a nanocrystal in which there is quantum confinement in all three dimensions (White, 2010).

Квантовые точки 7 могут содержать нанокристаллы, в которых имеется квантовая ловушка по всем трем измерениям (перевод ВОИС) (Уайт, 2010).

Термин quantum confinement образован посредством метафорического переноса на основе сходства функции. При переводе использована иная метафора - квантовая ловушка, хотя речь идет не о том, что для кван- тов ставятся некие ловушки: суть явления состоит в ограничении движения самих квантов по трем измерениям [Анищик и др., 2008].

Деметафоризация (5 лексических единиц).

(3) The invention relates to a self-healing and scratch resistant shape memory polymer system (Xie, 2010).

Изобретение относится к самовосстанавливающейся и устойчивой к царапинам полимерной системе с памятью формы (перевод ВОИС) (Кси, 2010).

Термин self-healing образован посредством метафорического переноса на основании сходства функции, однако в переводе наблюдается деметафоризация с калькированием морфологической структуры слова.

Метафоризация или деметафоризация (4 лексические единицы).

При переводе одного метафоризированного термина наблюдается использование и метафоризации, и деметафоризации.

(4) The sacrificial substrate includes a plurality of collagen fibers reinforced with a supporting material and intersecting with each other to form a network of collagen fibers having a plurality of openings (Locke, 2017).

Жертвенная подложка включает в себя множество коллагеновых волокон, армированных поддерживающим материалом и пересекающихся друг с другом с образованием сетки коллагеновых волокон, имеющей множество отверстий (перевод ВОИС) (Лок, 2017).

При переводе sacrificial возможна метафоризация с сохранением способа переноса значения по функции, что наблюдается при передаче на русский язык словосочетания sacrificial substrate как жертвенная подложка.

(5) The disclosed technology generally relates to forming a semiconductor structure and more particularly to forming a stack of layers of a semiconductor structure using a sacrificial layer that is removed during deposition of a functional layer (Swerts, 2016).

Раскрытая технология в общем относится к формированию полупроводниковой структуры и, в частности, к формированию пакета слоев полупроводниковой структуры с использованием временного слоя, который удаляется во время осаждения функционального слоя (перевод ВОИС) (Свертс, 2016). 
Компонент sacrificial в составе словосочетания sacrificial layer переводится как временный, следовательно, в переводе имеет место деметафоризация.

\section{Выводы}

В патентах как одном из жанров технического текста находит отражение информация о новейших открытиях и достижениях. Проведенное на материале патентов исследование показало, что метафорический перенос лежит в основе образования ряда ключевых терминов области наноматериалов и нанотехнологий, а метафоризированные термины передают когнитивную информацию. В переводе таких терминов на русский язык могут быть задействованы механизмы метафоризации, реметафоризации, деметафоризации, а также возможны переводы одного и того же термина с использованием как метафоризации, так и деметафоризации, что ведет к использованию в русском языке двух вариантов термина. При этом в рамках проведенного исследования можно говорить о меньшей представленности такого приема, как реметафоризация.

\section{СПИСОК ЛИТЕРАТУРЫ}

Алексеева И. С., 2008. Транслатологические типы текста. Опыт классификации. Ч. II // Вестник Санкт-Петербургского университета. Серия 9. Филология. Востоковедение. Журналистика. Вып. 3, ч. I. C. 32-40. URL: https://cyberleninka. $\mathrm{ru} /$ article/v/translatologicheskie-tipy-tekstaopyt-klassifikatsii-ch-ii (дата обращения: 11.02.2018).

Анищик В. М., Борисенко В. М., Жданов С. А., Толочко Н. К., Федосюк В. М., 2008. Наноматериалы и нанотехнологии / под ред. В. Е. Борисенко и Н. К. Толочко. Минск : Изд. центр БГУ. $372 \mathrm{c}$.

Виноградов В. В., 1980. О теории литературных стилей // Избранные труды. О языке художественной прозы. М. : Наука. С. 240-249.

Горохова Н. В., 2014. Метафоризация как процесс семантического развития терминов в дискурсе специалиста трубопроводного транспорта // Вестник Ленинградского государственного университета им. А.С. Пушкина. Вып. 6(102). C. 210-217. URL: http://cyberleninka.ru/article/n/ metaforizatsiya-kak-protsess-semanticheskogo- razvitiya-terminov-v-diskurse-spetsialistatruboprovodnogo-transporta.pdf (дата обращения: 11.02.2018).

Лакофф Дж., Джонсон М., 2004. Метафоры, которыми мы живем / пер. с англ. А. Н. Баранова, А. В. Морозова ; под ред. и с предисл. А. Н. Баранова. М. : Едиториал УРСС. 256 с.

Лосева О. М., 2015. Метафора в научно-техническом тексте // Филологические науки. Вопросы теории и практики. № 8 (50), ч. III. С. 118 121. URL: http://scjournal.ru/articles/issn_19972911_2015_8-3_34.pdf.

Уткина Т. И., 2006. Метафора в научно-популярном медицинском дискурсе: семиотический, когнитивно-коммуникативный, прагматический аспекты : дис. ... канд. филол. наук. Пермь, 2006. 210 c. URL: http://www.dissercat.com/content/ meta fora-v-nauchno-populyarnommeditsinskom-diskurse-semioticheskiikognitivno-kommun (дата обращения: 11.02.2018).

Шебеко Н. С., 2012. Метафора при переводе англоязычных нехудожественных текстов // Вестник Московского государственного лингвистическогоуниверситета. Вып. 17 (650). С. 60-71. URL: $\mathrm{http}: / /$ cyberleninka.ru/article/n/metafora-priperevode-angloyazychnyh-nehudozhestvennyhtekstov (дата обращения: 25.06.2017).

Finatto M. J. B., 2010. Metaphors in scientific and technical languages: challenges and perspective // Documentação de Estudos em Lingüística Teórica e Aplicada. Vol. 26. P. 645-656. URL: http:// www.scielo.br/scielo.php?script=sci arttext\& $\mathrm{pid}=\mathrm{S} 0102-44502010000300012$ (date of access: 28.08.2017).DOI: $10.1590 / \mathrm{S} 0102-44502010000300012$.

\section{ИСТОЧНИКИ И СЛОВАРИ}

Кси, 2010 - Кси Т., Ксяо Кс., Чен Ян Т. Самовосстанавливающаяся и устойчивая к царапинам полимерная система с памятью формы / пер. с англ. ВОИС // PATENTSCOPE. URL: https:// patentscope.wipo.int/search/en/detail.jsf? docId $=$ CN $83933205 \&$ recNum $=8 \&$ office $=$ \&queryString $=\mathrm{FP} \% 3 \mathrm{~A} \% 28$ graphene+selfhealing\%29\&prevFilter=\&sortOption=Pub+Date + Desc\&maxRec=9 (дата обращения: 27.06.2017). Ли, 2017 - Ли К., Хео Дж., Ю В., Шин Й. Графеновое электронное устройство с канальным слоем, включающим островки графена, а также способ его изготовления / пер. с англ. ВОИС // PATENTSCOPE. URL: https://patentscope.wipo. int/search/en/detail.jsf?docId=US192509690\& recNum $=1 \& \max R e c=7 \&$ office $=\&$ prevFilter $=$ $\&$ sortOption $=\mathrm{Pub}+$ Date + Desc\&queryString $=$ 
FP\%3A\%28\%2 2graphene+island\%22\%29\& tab=NationalBiblio(дата обращения: 27.06.2017).

Лок, 2017 - Локк К. Б., Робинсон Т. М., Кален Б. М. Пограничный слой, модулирующий протеазу, для использования при лечении ран отрицательным давлением / пер. с англ. ВОИС // PATENTSCOPE. URL: https://patentscope. wipo.int/search/en/detail.jsf?docId= EP199525481\&recNum $=15$ \&office $=$ \&query String $=$ FP\% 3A\%28sacrificial+layer $\% 29 \&$ prevFilter $=\&$ sortOption $=\mathrm{Pub}+$ Date + Desc \&max $\mathrm{Rec}=12823$ (дата обращения: 28.06.2017).

Свертс, 2016 - Свертс Дж., Мертенс С. Способ формирования пакета слоев с использованием временного слоя / пер. с англ. ВОИС // PATENTSCOPE. URL: https://patentscope. wipo.int/search/en/detail.jsf?docId= US177999988\&rec Num=7\&office $=$ \&query String=EN_ALLTXT\%3A\%28A + method + comprises+depositing $+\mathrm{a}+$ sacrificial + layer + \%29\&prevFilter $=\&$ sortOption=Relevance $\&$ $\operatorname{maxRec}=62773$ (дата обращения: 28.06.2017).

СЭСРЯ-Стилистический энциклопедический словарь русского языка / под ред. М. Н. Кожиной. 2-е изд., испр. и доп. М. : Флинта : Наука, 2006. 696 с.

Уaйm, 2010 - Уайт Р., Малик С., Ален М., Бейли М. Устройство и способ для восприятия анализируемого вещества с использованием графенового канала, квантовых точек и электромагнитного излучения / пер. с англ. ВОИС // PATENTSCOPE. URL: https://patentscope. wipo.int/search/en/detail.jsf?docId= WO2017072399\& recNum $=8 \& \max R e c=17452 \&$ office $=\&$ prevFilter $=\&$ sortOption $=$ Pub + Date + Desc \& queryString $=\mathrm{FP} \% 3 \mathrm{~A} \% 28$ graphene $\%$ $29 \& \mathrm{tab}=$ PCTDescription (дата обращения: 27.06.2017).

Lee, 2017 - Lee K., Heo J., Yu W., Shin Y. Graphene electronic device having channel layer including graphene islands and method of fabricating the same // PATENTSCOPE. URL: https:// patentscope.wipo.int/search/en/detail.jsf? docId=US192509690\& recNum=1\&maxRec= $7 \&$ office $=\&$ prevFilter $=\&$ sortOption $=\mathrm{Pub}+$ Date + Desc \&queryString $=\mathrm{FP} \% 3 \mathrm{~A} \% 28 \% 22$ graphene + island $\% 22 \% 29 \&$ tab $=$ NationalBiblio (date of access: 27.06.2017).

Locke, 2017 - Locke Chr. Br., Robinson T. M., Cullen B. M. Protease modulating wound interface layer for use with negative pressure wound therapy // PATENTSCOPE. URL: https:/ /patentscope. wipo.int/search/en/detail.jsf? docId $=$ EP199525481\&recNum $=15$ \&office $=\&$ query String $=\mathrm{FP} \% 3 \mathrm{~A} \% 28$ sacrificial + layer $\%$ 29\&prev Filter $=\&$ sortOption $=$ Pub + Date + Desc $\&$ $\operatorname{maxRec}=12823$ (date of access: 28.06.2017).
Swerts, 2016-Swerts J., Mertens S. Method of forming a stack of layers using a sacrificial layer // PATENTSCOPE. URL: https://patentscope. wipo.int/search/en/detail.jsf?docId= US $177999988 \&$ rec Num $=7$ \& office $=$ \&queryString $=\mathrm{EN}$ _ALLTXT $\% 3 \mathrm{~A} \% 28 \mathrm{~A}+$ method + comprises + depositing $+\mathrm{a}+$ sacrificial + layer $+\% 29 \&$ prevFilter $=\&$ sortOption $=$ Relevance\&maxRec $=62773$ (date of access: 28.06.2017).

Xie, 2010-Xie T., Xiao X., Cheng Yang T. Self-healing and scratch resistant shape memory polymer system // PATENTSCOPE. URL: https:// patentscope.wipo.int/search/en/detail.jsf? docId $=$ CN83933205\&rec Num $=8$ \&office $=$ \&queryString $=\mathrm{FP} \% 3 \mathrm{~A} \% 28$ graphene + selfhealing\%29\&prevFilter $=\&$ sortOption $=\mathrm{Pub}+$ Date + Desc\&maxRec $=9$ (date of access: 27.06.2017).

White, 2010 - White R., Malik S., Allen M., Bailey M. Apparatus and method for sensing an analyte, using a graphene channel, quantum dots and electromagnetic radiation // PATENTSCOPE. URL: https://patentscope.wipo.int/search/en/ detail.jsf?docId=WO2017072399\&recNum= $8 \&$ maxRec $=1$ 7452\&office $=$ \&prevFilter $=\&$ sort Option $=$ Pub + Date + Desc\&queryString $=\mathrm{FP} \% 3 \mathrm{~A} \% 28$ graphene $\% 29 \&$ tab $=$ PCTDescription (date of access: 27.06.2017).

\section{REFERENCES}

Alekseeva I.S., 2008. Translatological text types. A classification. Part II. Vestnik SanktPeterburgskogo universiteta. Seriya 9. Filologija. Vostokovedenie. Zhurnalistika [Vestnik of Saint Petersburg University. Series 9. Philology. Oriental Studies. Journalism], iss. 3, part I, pp. 32-40. URL: https://cyberleninka.ru/ article/v/translatologicheskie-tipy-teksta-opytklassifikatsii-ch-ii (accessed February 11, 2018).

Anishchik V.M., 2008. Nanomaterials and nanotechnology. Minsk, BSUPublishing Center. 372 p.

Vinogradov V.V., 1980. On the theory of literature styles. Izbrannye trudy. O yazyke khudozhestvennoy prozy [Selected Works. On the Language of Fiction]. Moscow, Nauka Publ., pp. 240-249.

Gorokhova N.V., 2014. Metaphorical meaning formation as a process of terms' semantic development in the discourse of pipeline transport specialist. Vestnik Leningradskogo gosudarstvennogo universiteta im. A.S. Pushkina [Vestnik of Pushkin Leningrad State University], iss. 6(102), pp. 210-217. URL: http://cyberleninka.ru/article/ $\mathrm{n} / \mathrm{m}$ et a forizat s i y - kak-protsess - 
semanticheskogo-razvitiya-terminov-vdiskurse-spetsialista-truboprovodnogotransporta.pdf (accessed February 11, 2018).

Lakoff G., Johnson M., 2004. Metaphors we live by. Moscow, Editorial URSS. 256 p.

Loseva O.M., 2015. The metaphor in the scientifictechnical text. Filologicheskie nauki. Voprosy teorii i praktiki [Philological Sciences. Issues of theory and practice], no. 8 (50), part III, pp. 118-121. URL: http://scjournal.ru/articles/ issn_1997-2911_2015_8-3_34.pdf.

Utkina T.I. 2006 . The metaphor in the popularscientific medical discourse: semiotic, cognitive-communicative, pragmatic aspects. Dissertation for Candidate of Sciences in Philology Degree. Perm, PGU. 210 p. URL: http:// www.dissercat.com/content/metafora-vnauchno-populyarnom-meditsinskom-diskursesemioticheskii-kognitivno-kommun (accessed 11 February 2018).

Shebeko N.S., 2012. The metaphor and the translation of English non-fiction texts. Vestnik Moskovskogo gosudarstvennogo lingvisticheskogo universiteta [MSLU's Vestnik], iss. 17 (650), pp. 60-71. URL: http://cyberleninka.ru/article/n/metafora-priperevode-angloyazychnyh-nehudozhestvennyhtekstov (accessed 25 June 2017).

Finatto M.J.B., 2010. Metaphors in scientific and technical languages: challenges and perspective. Documentação de Estudos em Lingüística Teórica e Aplicada, vol. 26, pp. 645656. URL: http://www.scielo.br/scielo.php? script $=$ sci arttext\&pid $=\mathrm{S} 0102-445020$ 10000300012 (accessed 28 August 2017). DOI: 10.1590/S0102-44502010000300012.

\section{SOURCES AND DICTIONARIES}

Xie T., Xiao X., Cheng Yang T. Samovosstanavlivayushchayasya $i$ ustoychivaya $k$ tsarapinam polimernaya sistema s pamyatyu formy [SelfHealing and Scratch Resistant Shape Memory Polymer System]. PATENTSCOPE. URL: https:/ /patentscope. wipo.int/search/en/detail.jsf? docId $=$ CN83933205\&recNum $=8$ \&office $=\&$ queryString $=\mathrm{FP} \% 3 \mathrm{~A} \%$ 28graphene + selfhealing\%29\& prevFilter= \&sortOption= Pub + Date + Desc \&maxRec $=9$ (accessed 27 June 2017).

Lee K., Heo J., Yu W., Shin Y. Grafenovoe elektronnoe ustroystvo s kanalnym sloem, vklyuchayushchim ostrovki grafena, a takzhe sposob ego izgotovleniya [Graphene Electronic Device Having Channel Layer Including Graphene Islands, and the Method of Its Production].
PATENTSCOPE. URL: https://patentscope. wipo.int/search/en/detail.jsf?docId=US192509690 \&recNum $=1 \&$ maxRec $=7$ \&office $=$ \&prevFilter $=$ \&sortOption $=\mathrm{Pub}+$ Date + Desc\&queryString $=\mathrm{FP} \%$ $3 \mathrm{~A} \% 28 \% 22$ graphene + island $\% 22 \% 29 \& \mathrm{tab}=$ NationalBiblio (accessed 27 June 2017).

Locke Chr. Br., Robinson T.M., Cullen B.M. Pogranichnyy sloy, moduliruyushchiy proteazu, dlya ispolzovaniya pri lechenii ran otritsatelnym davleniem [Protease Modulating Wound Interface Layer for Use with Negative Pressure Wound Therapy]. PATENTSCOPE. URL: https:// patentscope.wipo.int/search/en/detail.jsf? docId $=$ EP $199525481 \&$ recNum $=15 \&$ office $=$ \&queryString $=\mathrm{FP} \% 3 \mathrm{~A} \% 28$ sacrificial + layer $\%$ $29 \&$ prevFilter $=\&$ sortOption $=\mathrm{Pub}+$ Date + Desc\&maxRec=12823 (accessed 28 June 2017).

Swerts J., Mertens S. Sposob formirovaniya paketa sloev s ispolzovaniem vremennogo sloya [Method of Forming a Stack of Layers Using a Sacrificial Layer]. PATENTSCOPE. URL: https:// patentscope.wipo.int/search/en/detail.jsf? docId=US177 999988\&recNum=7\&office= \&queryString $=$ EN_ALLTXT $\% 3 \mathrm{~A} \% 28 \mathrm{~A}+$ method + comprises + depositing $+\mathrm{a}+$ sacrificial + layer + $\% 29 \&$ prevFilter $=\&$ sortOption $=$ Relevance $\&$ maxRec $=62773$ (accessed 28 June 2017).

Kozhina M.N., ed. 2006. Stylistic encyclopedic dictionary of the Russian language. Moscow, Flinta; Nauka Publ. 696 p.

White R., Malik S., Allen M., Bailey M. Ustroystvo i sposob dlya vospriyatiya analiziruemogo veshchestva $\mathrm{s}$ ispolzovaniem grafenovogo kanala, kvantovykh tochek i elektromagnitnogo izlucheniya [Apparatus and Method for Sensing an Analyte, Using a Graphene Channel, Quantum Dots and Electromagnetic Radiation]. PATENTSCOPE. URL: https://patentscope. wipo.int/search/en/detail.jsf?docId= WO2017072399\& recNum $=8 \& \max R e c=17452 \&$ office $=\&$ prevFilter $=\&$ sortOption $=\mathrm{Pub}+$ Date + Desc\&queryString=FP\%3A\%28graphene $\% 29$ $\&$ tab $=$ PCTDescription (accessed 27 June 2017).

Lee K., Heo J., Yu W., Shin Y. Graphene electronic device having channel layer including graphene islands and method of fabricating the same. PATENTSCOPE. URL: https://patentscope. wipo.int/search/en/detail.jsf?docId= US $192509690 \& \mathrm{recNum}=1 \& \max \mathrm{Rec}=7 \&$ office $=\&$ prevFilter $=\&$ sortOption $=\mathrm{Pub}+$ Date + Desc\&queryString $=\mathrm{FP} \% 3 \mathrm{~A} \% 28 \% 22$ graphene+ island $\% 22 \% 29 \&$ tab $=$ NationalBiblio (accessed 27 June 2017).

Locke Chr. Br., Robinson T. M., Cullen B. M. Protease modulating wound interface layer for use with negative pressure wound therapy. 
PATENTSCOPE. URL: https://patentscope. wipo.int/search/en/detail.jsf?docId= EP $199525481 \&$ rec Num= 15 \& office $=$ \&queryString $=\mathrm{FP} \% 3 \mathrm{~A} \% 28$ sacrificial + layer $\%$ $29 \&$ prevFilter $=\&$ sortOption $=\mathrm{Pub}+$ Date + Desc\&maxRec $=12823$ (accessed 28 June 2017).

Swerts J., Mertens S. Method of forming a stack of layers using a sacrificial layer. PATENTSCOPE. URL: https://patentscope.wipo.int/search/en/ detail.jsf?docId $=$ US177999988\&recNum $=7 \&$ office $=\&$ queryString=EN_ALLTXT\%3 A\%28A+ method + comprises + depositing $+\mathrm{a}+$ sacrificial + layer $+\% 29 \&$ prevFilter $=\&$ sortOption $=$ Relevance $\& \operatorname{maxRec}=62773$ (accessed 28 June 2017).

White R., Malik S., Allen M., Bailey M. Apparatus and method for sensing an analyte, using a graphene channel, quantum dots and electromagnetic radiation. PATENTSCOPE. URL: https:// patentscope.wipo.int/search/en/ detail.jsf?docId $=$ WO2017072399\&recNum $=8 \&$ $\max \operatorname{Rec}=17452 \&$ office $=\&$ prevFilter $=$ \&sortOption $=\mathrm{P}$ ub+Date + Desc\&queryString $=$ FP\%3A\%28graphene\%29\&tab=PCTDescription (accessed 27 June 2017).

Xie T., Xiao X., Cheng Yang T. Self-healing and scratch resistant shape memory polymer system. PATENTSCOPE. URL: https://patentscope. wipo.int/search/en/detail.jsf?docId=CN83933205 \&recNum $=8 \&$ office $=\&$ queryString $=\mathrm{FP} \% 3 \mathrm{~A} \%$ 28 graphene+selfhealing $\% 29 \&$ prevFilter $=$ \&sortOption $=\mathrm{Pub}+$ Date + Desc\&max Rec $=9$ (accessed 27 June 2017).

\section{Information about the Author}

Natalya V. Sokolova, Candidate of Sciences (Philology), Associate Professor, Department of Foreign Languages, Linguistics and Translation, Perm National Research Polytechnic University, Prosp. Komsomolsky, 29, 614990 Perm, Russia, ntlk0@mail.ru, https://orcid.org/0000-0003-0550-2272

\section{Информация об авторе}

Наталья Владимировна Соколова, кандидат филологических наук, доцент кафедры иностранных языков, лингвистики и перевода, Пермский национальный исследовательский политехнический университет, просп. Комсомольский, 29, 614990 г. Пермь, Россия, ntlk0@mail.ru, https:// orcid.org/0000-0003-0550-2272 